\title{
Análise da decisão de entrega de resíduos de equipamentos elétricos e eletrónicos em Portugal
}

\author{
Carla Ferreira, Lígia M. Costa Pinto* \\ NIMA, Escola de Economia e Gestão, Universidade do Minho, \\ 4710-057 Braga, Portugal
}

\section{RESUMO}

O presente artigo tem como objetivo analisar os determinantes da decisão de entrega dos Equipamentos Elétricos e Eletrónicos em fim de vida para reciclagem. Desta forma, pretende-se avaliar as motivações e interesses dos utilizadores destes equipamentos em relação ao mercado de retoma dos Equipamentos Elétricos e Eletrónicos em Fim de Vida. Os resultados obtidos indicam que existe uma falta de sensibilidade dos consumidores para o processo de retoma dos seus equipamentos. Por outro lado, mostram que as condições de informação e as características sociodemográficas interferem nos desejos dos consumidores para a devolução dos seus Resíduos de Equipamentos Elétricos e Eletrónicos. Neste âmbito, pode-se concluir que o mercado de retoma, de Equipamento Elétricos e Eletrónicos em Fim de Vida em Portugal, encontra-se em fase de crescimento, nomeadamente, ao nível de estratégias de retoma e competitividade.

Palavras-Chave - Incentivos de retoma; Resíduos de equipamentos elétricos e eletrónicos; Reciclagem; Economia circular.

doi: $10.22181 /$ aer.2016.0101

\footnotetext{
* Autor para correspondência

E-mail: ligiacpinto@gmail.com (Prof. L. Pinto)
} 


\title{
Analysis of take-back decisions regarding electrical and electronic equipment waste in Portugal
}

\author{
Carla Ferreira, Lígia M. Costa Pinto* \\ ${ }^{a}$ NIMA, Escola de Economia e Gestão, Universidade do Minho, \\ 4710-057 Braga, Portugal
}

\section{ABSTRACT}

This paper aims to investigate the determinants of consumers' decision to take-back Electrical and Electronic Equipment Waste in Portugal. It assesses users' perception of these items, evaluating the motivations and interests they have concerning the market of electrical and electronic equipment waste. Results indicate a lack of awareness by consumers about the process of take-back available for their equipment. On the other hand, results show that information conditions and sociodemographic factors affect consumers' motivations for returning the electrical and electronic equipment at the end of life. In this context, it can be concluded that the Portuguese market for the recovery of Electrical and Electronic Equipment Waste is still in its infancy, particularly in terms of recovery strategies and competitiveness.

Keywords - Take-back Incentives; electrical and electronic equipment waste; recycling; circular economy.

doi: $10.22181 /$ aer.2016.0101

\footnotetext{
* Corresponding author

E-mail: ligiacpinto@gmail.com (Prof. L. Pinto)
} 
O avanço tecnológico, aliado ao rápido crescimento económico, proporcionou um aumento significativo da comercialização de Equipamentos Elétricos e Eletrónicos (EEE), bem como a redução do tempo de vida útil dos mesmos. Em consequência, este avanço tem vindo a gerar a produção de cada vez maiores quantidades de Resíduos de Equipamentos Elétricos e Eletrónicos (REEE). Só na União Europeia estima-se que em 2020 sejam produzidos aproximadamente 12,3 milhões de toneladas (Mt) destes resíduos (Lundgren 2012).

A diversidade de tipologia e uso de EEE acarreta uma variedade enorme de REEE, o que por sua vez não permite uma definição específica e coerente de REEE, motivo pelo qual existem, atualmente, várias definições para estes na literatura específica, muitas delas pouco consensuais entre vários autores e/ou entidades intervenientes (Widmer et al. 2005). Com o objetivo de agrupar os REEE, segundo a sua composição e funcionalidade, a Diretiva propõe a classificação dos EEE em dez categorias distintas: Grandes eletrodomésticos; Pequenos eletrodomésticos; Equipamentos informáticos e de telecomunicações (IT); Equipamentos de consumo; Equipamentos de Iluminação; Ferramentas Elétricas e Eletrónicas; Brinquedos e Equipamentos de desporto e lazer; Aparelhos médicos; Instrumentos de Monitorização e Controlo e Distribuidores automáticos.

A introdução da nova Diretiva - Diretiva n. ${ }^{0}$ 2012/19/EU - levou à redefinição do conceito e categorização dos EEE, uma vez que todos os EEE se encontram abrangidos pela mesma. Porém, estas alterações encontram-se em fase de transição, uma vez que a partir de 15 de agosto de 2018 todos os dispositivos eletrónicos devem ser agrupados em uma de seis novas categorias. Para além destas alterações relevantes, também as metas de recolha foram reformuladas, sendo calculadas com base na quantidade de EEE colocados no mercado nos três anos anteriores, o que equivale a uma recolha de, aproximadamente, $10 \mathrm{Mt}$ (sensivelmente $20 \mathrm{~kg}$ per capita), ou seja uma meta cinco vezes superior à meta atual dos $4 \mathrm{~kg}$ per capita estabelecida na Diretiva n.0 2012/19/EU (Marçal 2013).

A deposição de EEE em Fim de Vida tem vindo a aumentar, levando a graves problemas ambientais e económicos (Lundgren 2012). Os principais riscos da má gestão dos REEE para a saúde pública e o ambiente prendem-se essencialmente com os metais pesados e outras substâncias orgânicas poluentes que se encontram nesta tipologia de resíduos (Lundgren 2012). Ao nível económico, os EEE em Fim de Vida apresentam na sua constituição materiais valiosos e escassos que Ihe conferem algum valor económico. Este facto tem proporcionado espaço para a criação de um negócio: a exportação de REEE à escala global, especialmente para economias em desenvolvimento.

O presente trabalho foi desenvolvido no âmbito de uma tese de mestrado (Ferreira 2015) e tem como objetivo analisar os determinantes da decisão de entrega dos Equipamentos Elétricos e Eletrónicos em fim de vida para reciclagem. Desta forma, pretende-se avaliar as motivações e interesses dos utilizadores destes equipamentos em relação ao mercado de retoma dos Equipamentos Elétricos e Eletrónicos em Fim de Vida. 
O crescimento económico, aliado à evolução tecnológica dos últimos anos, tem levado à produção de grandes quantidades de resíduos de diversas tipologias, o que impulsionou a procura de soluções eficazes para o Fim de Vida de cada produto (Gui et al. 2013). Neste seguimento, surge o conceito RAP que consiste numa abordagem político-ambiental na qual a responsabilidade do produtor se estende à fase pós-consumo do ciclo de vida dos produtos por si colocados no mercado. O principal objetivo da RAP é a transferência da responsabilidade financeira ou física de recolha e valorização dos equipamentos em Fim de Vida para o produtor, de modo a que este proporcione um adequado encaminhamento e tratamento, formulando, para isso, leis de retoma dos produtos. Este conceito visa não só reduzir os impactes negativos dos resíduos, mas também promover a criação de incentivos para o design de produtos mais fáceis de recuperar e reutilizar, integrando a lógica de economia circular quer no design quer no processo de gestão dos materiais que compõem o produto (Atasu e Subramanian 2012).

Teoricamente a RAP é uma obrigação individual de cada produtor. Porém, na prática, os produtores exercem esta obrigação de forma coletiva. Por consequência, esta obrigatoriedade leva à criação de dois tipos de mercado de retoma de resíduos: monopólio e concorrencial (EC 2014). Num sistema monopolista de RAP, verifica-se a existência de uma organização única que absorve a responsabilidade do produtor (da sigla em inglês PRO), que aplica o princípio RAP em nome dos produtores. Assim, os produtores pagam um custo médio de reciclagem, de acordo com a quantidade de produtos colocados no mercado, ficando impedidos de optar pela solução mais competitiva (Carvalho 2008). Em contrapartida, num sistema concorrencial existe uma entidade nacional que supervisiona todo o processo, designada por Clearing House National (CHN), na qual os produtores ficam sujeitos a reportar a quantidade de produtos colocados no mercado, para o cálculo da respetiva quota de mercado e da quantidade de REEE a gerir. De acordo com este modelo de implementação da RAP, os produtores são livres de contratualizar os serviços de recolha e reciclagem através dos General Contractors (GC), de forma a alcançar as quantidades que the foram atribuídas a um menor custo (Carvalho 2008).

A determinação de qual o sistema de retoma mais adequado para a implementação da RAP nos EEE em Fim de Vida, tendo em conta a sua eficácia técnica e económica, não é consensual e depende de vários fatores. De acordo com os resultados de Toyasaki et al. (2009), um regime de retoma competitivo torna-se mais favorável para as partes interessadas, uma vez que os recicladores têm um maior incentivo para praticar taxas de reciclagem inferiores, o que leva os fabricantes a auferirem lucros superiores tendo, desta forma, margem para reduzir o preço final dos produtos. Porém, o benefício generalizado para todos os stakeholders do mercado depende do grau de substituibilidade dos produtos $(\beta)$, ou seja, só existe um ganho genérico se o grau de substituibilidade dos produtos for relativamente baixo. Caso contrário, os recicladores preferem operar sob a tutela de uma organização sem fins lucrativos, em regime de retoma monopolista (Toyasaki et al. 2009).

Apesar da responsabilidade de financiamento se encontrar do lado do produtor, sendo estes os stakeholders principais do mercado, vários outros agentes interagem no mercado com interesses por vezes distintos. Entre estes encontram-se os operadores de resíduos; autoridades nacionais; PRO; 
retalhistas e autarquias (Kalimo et al. 2014). O consumidor (proprietário do equipamento) desempenha um papel fundamental em toda a estrutura de mercado, visto que é responsável pelo fornecimento da matéria-prima ao mercado de recolha e reciclagem, sendo também chamado a co-financiar a fase de pós-consumo dos EEE através do preço final dos equipamentos (Kunz et al. 2013).

Na União Europeia, a estrutura de mercado de REEE varia de acordo com as características económicas e sociais de cada Estado-Membro. Não existe, portanto, uma estrutura de mercado transversal, uma vez que co-existem diferentes métodos de recolha e financiamento dentro da Europa (UNEP 2012). Alguns Estados-Membros, como por exemplo a Bélgica, optaram por sistemas monopolistas, em que uma única PRO é responsável por todo o mercado de recolha, comunicação e encaminhamento dos REEE (UNEP 2012). Por outro lado, países como Portugal e Alemanha fomentam um mercado concorrencial, através do licenciamento de várias PRO (UNEP 2012). A nível nacional estão licenciadas duas entidades gestoras (AMB3E e ERP PT), para as quais os produtores transferem a responsabilidade de recolha $e$ processamento dos seus EEE em Fim de Vida, através de uma prestação financeira (Niza et al. 2014). Com o objetivo de supervisionar todo o mercado, os produtores ficam sujeitos a um registo de produção na ANREEE (Associação Nacional de Resíduos de Equipamentos Elétricos e Eletrónicos) para que, posteriormente, se estabeleçam as taxas de recolha de cada produtor, bem como a verificação do cumprimento da RAP (Niza et al. 2014).

Em 2012 Portugal apresentou uma taxa de recolha de 3,9 kg ReEE/habitante. Um valor aquém do verificado na União Europeia de $6,9 \mathrm{~kg}$ REEE/habitante, fruto de um mercado ainda em expansão, com diversas barreiras ao nível dos operadores de resíduos e logística de funcionamento das PRO. Apesar do mercado nacional se apresentar como um mercado concorrencial, não se verificam diferenças significativas ao nível dos custos imputados ao produtor nem ao nível dos incentivos aos consumidores de forma a angariar mais quantidades de REEE (Niza et al. 2014).

Os hábitos dos consumidores, em relação ao destino dado aos seus EEE em Fim de Vida, dependem da peculiaridade de cada país, dado que as características da sociedade influenciam a forma como os indivíduos se envolvem nos processos de recolha e reciclagem dos REEE. Neste sentido, vários estudos têm vindo a ser desenvolvidos, com o objetivo de averiguar os fatores que determinam o comportamento dos consumidores para a entrega dos REEE. Yin et al. (2013) concluíram que os principais fatores que afetam a perceção dos consumidores para a entrega dos telemóveis em Fim de Vida são a localização geográfica, o nível de escolaridade e o rendimento mensal.

Wang et al. (2011) estudaram as variáveis intrínsecas ao comportamento dos residentes de Pequim, no que respeita à vontade de participar no processo de retoma e reciclagem dos REEE. Os resultados obtidos indicam que os indivíduos apresentam pouca predisposição para procederem à devolução dos seus EEE em Fim de Vida. De entre os fatores que mais impulsionam a entrega de EEE em fim de vida, Wang et al (2011) identificaram os hábitos de reciclagem, os benefícios económicos e a proximidade a locais de entrega do velho equipamento. Pelo contrário, o estudo conclui que fatores como o nível de escolaridade e o rendimento mensal não determinam o comportamento dos utilizadores na gestão dos REEE. 
A análise dos determinantes da decisão de entrega de EEE em fim de vida desenvolveu-se através do estudo da perceção dos consumidores, em relação ao processo de retoma dos REEE. Neste sentido, seguiu-se uma pesquisa quantitativa, com a construção e aplicação de um questionário a utilizadores de EEE. Este questionário é constituído por questões fechadas, semiabertas e de opinião, perfazendo um total de doze questões, das quais quatro correspondem à caracterização sociodemográfica da amostra.

A distribuição e disponibilização do inquérito a utilizadores de EEE realizou-se por via eletrónica, através do preenchimento e envio on-line do questionário, entre janeiro e julho de 2015. A amostra obtida é constituída por 145 indivíduos. No entanto, face ao meio de divulgação do inquérito, é expectável uma amostra com um nível educacional elevado, uma menor variabilidade de idades e uma maior familiaridade com equipamentos eletrónicos. De acordo com os objetivos propostos, o problema de pesquisa passa por:

- Identificar os fatores sociodemográficos que influenciam significativamente o comportamento dos consumidores na retoma dos seus EEE em Fim de Vida;

- Identificar as motivações e comportamentos que influenciam o mercado de retoma de REEE.

Para além da análise descritiva, os dados recolhidos são utilizados para estimação de um modelo estatístico multivariado. No modelo estimado, a variável dependente assume o valor um se o indivíduo diz entregar o seu EEE em fim de vida para reciclagem, e o valor zero caso contrário. As variáveis independentes são o Nível de escolaridade; Rendimento mensal; Idade; Hábitos de recuperação dos REEE e Conhecimento de campanhas de retoma de REEE. Dada a natureza binária da variável dependente, o modelo escolhido é o modelo probit estimado através do método de máxima verosimilhança. Os efeitos marginais de cada variável independente sobre a probabilidade da variável dependente tomar o valor unitário são usados para testar as seguintes hipóteses de pesquisa:

H1 - As variáveis sociodemográficas são cruciais para o processo de retoma dos EEE em Fim de Vida;

H2 - O conhecimento das campanhas de retoma afetam o comportamento de entrega dos REE dos consumidores;

H3 - Os hábitos de recuperação dos equipamentos explicam a decisão dos consumidores entregarem os REEE.

\section{RESULTADOS E DISCUSSÃO}

Do total da amostra $(\mathrm{N}=145)$, mais de metade dos inquiridos é do sexo feminino $(68 \%)$ e $40 \%$ possui o grau académico de mestrado. No que diz respeito à ocupação corrente dos participantes, $64 \%$ dos indivíduos são trabalhadores por conta de outrem. O rendimento familiar líquido mensal médio, auferido pelos participantes, é de $1659 €$ (desvio padrão de 932€).

De acordo com a análise descritiva, os equipamentos que apresentam um tempo de utilização superior a 10 anos, com uma frequência de respostas igual ou superior a 50\% são: "Frigorifico"; Máquina de Lavar"; " Micro-ondas" e "Televisão/LCD". Em contrapartida, os equipamentos da categoria 3, como 
o "Telemóvel" e o " Computador", apresentam um tempo de utilização de 3 a 5 anos, sendo que a frequência de respostas se encontra próxima dos $50 \%$. Estes resultados, por sua vez, encontram-se em linha com os dados teóricos, ou seja, os consumidores substituem mais rapidamente os equipamentos informáticos e de telecomunicações de uso individual, comparativamente aos grandes equipamentos (Premalatha et al. 2015).

No que respeita aos hábitos na fase pós-consumo dos EEE, verifica-se que mais de metade dos inquiridos se dirige a um ponto de recolha $(66 \%)$; destes, $89 \%$ encaminham os seus REEE para uma recolha de proximidade. Existe um leque de motivos que levam os consumidores a não utilizarem os pontos de recolha de REEE. O principal motivo relaciona-se com o desconhecimento dos pontos de recolha (60\% dos inquiridos) e é seguido da distância entre a residência e os pontos de recolha ( $32 \%$ dos inquiridos).

Os resultados obtidos apontam ainda para alguma ineficácia das campanhas de incentivo à retoma implementadas em Portugal. Nomeadamente, na opinião dos inquiridos, estas campanhas não criam incentivos suficientes que levem os utilizadores a transportarem os seus EEE em Fim de Vida até a um local de entrega, como seja um retalhista. Assim, constata-se que $64 \%$ dos inquiridos não conhece qualquer campanha de incentivo à retoma, sendo que apenas $36 \%$ do total da amostra conhece algum tipo de campanha. De entre os que conhecem as campanhas, só uma pequena percentagem de indivíduos (15\%) já usufruiu desses mesmos incentivos.

Com o objetivo de investigar o tipo de incentivos desejados pelos consumidores de EEE, procedeu-se à análise da aceitabilidade de cinco esquemas de incentivos diferentes. Os esquemas de incentivos considerados foram: incentivos fiscais, troca por novo, recolha em casa, cupão de desconto, troca por dinheiro. A Figura 1 ilustra o nível de preferência (1menos preferido a 5 - mais preferido) dos inquiridos, para cada um dos cinco incentivos.

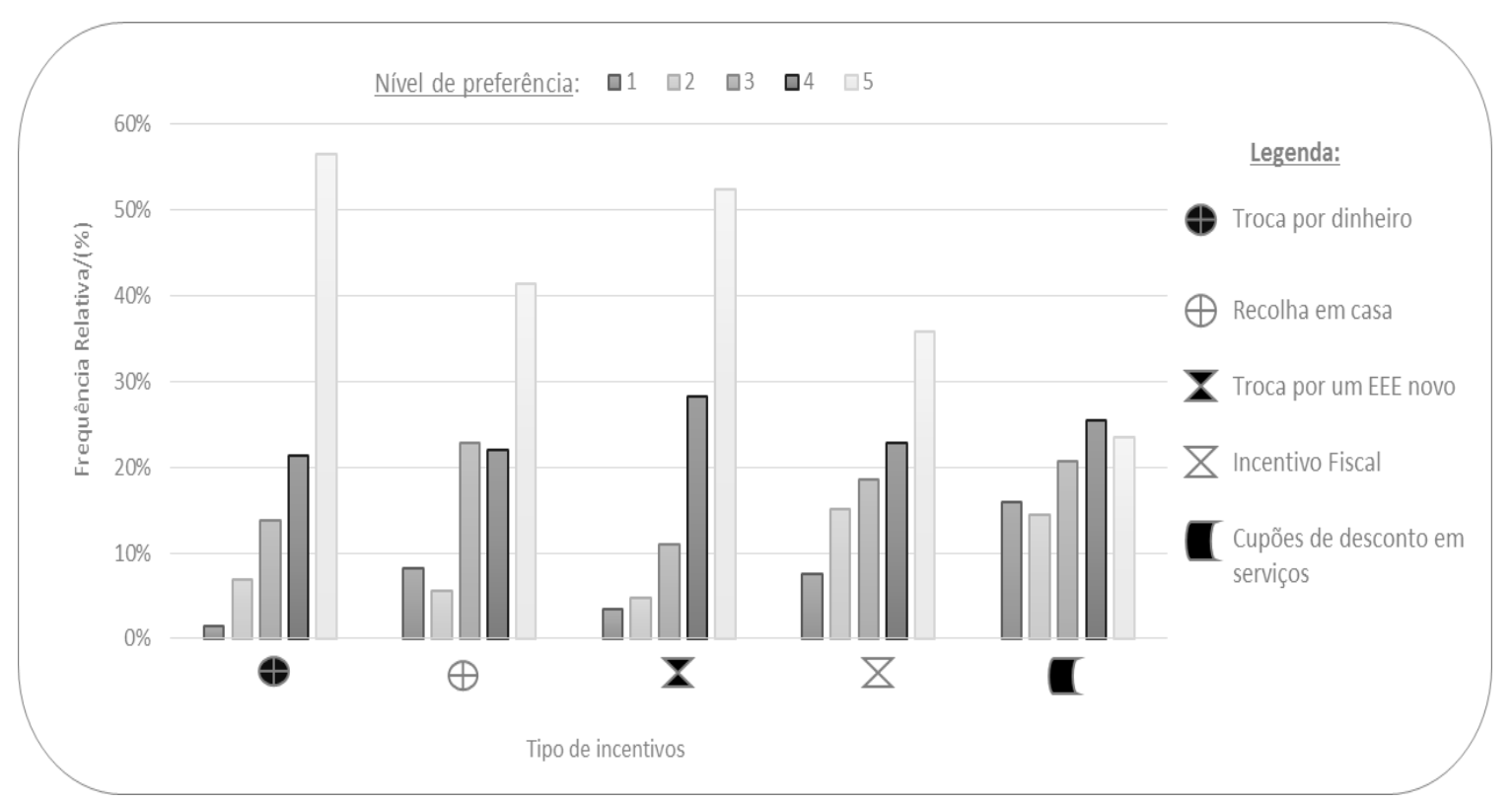

Figura 1. Nível de aceitabilidade dos inquiridos em relação aos cinco esquemas de incentivo em estudo ( 1 - menos preferido; 5 - mais preferido) 
Face aos resultados obtidos (Figura 1 ), verifica-se que os cinco incentivos proposta são, de uma forma geral, bem aceites pelos indivíduos. Comparando o grau de preferência dos participantes, os incentivos preferidos são: " Troca do EEE em Fim de Vida por dinheiro" (57\%) e a "Troca por EEE novo" (52\%). Em contrapartida, a troca por " Cupões de Descontos em Serviços" é o incentivo menos valorizado pelos inquiridos (23\%). Note-se que a recolha em casa do REEE não é considerada muito relevante para os indivíduos, que privilegiam a retoma por um valor económico ou um novo equipamento.

Apesar de a análise da aceitabilidade dos diferentes tipos de campanhas prover implicações interessantes, uma análise dos determinantes da decisão de entrega dos EEE em fim de vida permitirá identificar quais as caraterísticas dos utilizadores que, independentemente da campanha específica em causa, com maior probabilidade entregam os seus EEE em fim de vida para reciclagem ou reutilização. O Quadro 1 reporta os resultados (efeitos marginais) da estimação do modelo PROBIT que permitirá testar se as variáveis sociodemográficas, o conhecimento de campanhas e os hábitos de recuperação dos EEE determinam de forma estatisticamente significativa a probabilidade de entrega dos EEE em Fim de Vida. Pela análise da mesma, verifica-se que apenas a variável idade é estatisticamente significativa (para um nível de significância de $1 \%$ ). Isto é, de acordo com os resultados, a probabilidade dos utilizadores procederem à entrega dos seus REEE aumenta 1,81 pontos percentuais por cada ano de idade do indivíduo. No que diz respeito às restantes variáveis independentes, não existe evidência estatística suficiente para concluir pela sua influência sobre a probabilidade de entrega dos REEE. Assim, para a hipótese H1, os resultados encontram-se em concordância com os encontrados no estudo de Wang et al. (2011). Paralelamente, não foram encontradas evidências que suportem as hipóteses $\mathrm{H} 2 \mathrm{e} \mathrm{H} 3$.

Quadro 1. Efeitos marginais do modelo PROBIT em relação à $\mathrm{H} 1, \mathrm{H} 2$ e H3

\begin{tabular}{ccc}
\hline Variáveis independentes & Efeitos Marginais & Desvio-Padrão \\
\hline Escolaridade & 0,0287 & 0,0302 \\
Género & 0,1009 & 0,0422 \\
Rendimento-mensal & $-0,0001$ & $0,4 \times 10^{-4}$ \\
Idade & $0,0181^{*}$ & 0,0049 \\
Conhece-campanha & 0,0943 & 0,0896 \\
Recupera-EEE & $-0,0118$ & 0,1883 \\
\hline
\end{tabular}

*significância 1\%. N= 143. Log pseudolikelihood= $-77,62$. Probabilidade prevista $=71 \%$

\section{CONCLUSÕES}

Face aos avanços tecnológicos e hábitos de consumo, a boa gestão dos REEE tornou-se imprescindível e uma das principais preocupações ao nível das políticas ambientais na Europa. Neste sentido, a investigação levada a cabo no presente estudo permite retirar as seguintes conclusões, relativamente ao mercado de retoma de REEE em Portugal:

- Existem lacunas na criação e divulgação das campanhas de incentivo à retoma dos EEE em Fim de Vida. Verifica-se que conhecer as campanhas de retoma em vigor não aumenta a disposição dos indivíduos para a entrega; 
- A probabilidade de entrega de EEE em fim de vida é explicada fundamentalmente pela idade; contudo, no seu conjunto, as variáveis sociodemográficas consideradas explicam de forma estatisticamente significativa a decisão de entrega dos EEE.

- Os hábitos de recuperação de EEE, nomeadamente de grandes equipamentos, não influenciam a escolha dos incentivos por parte dos utilizadores. O incentivo económico "Troca por dinheiro" seria, de acordo com os resultados deste estudo, o mais promissor.

Em suma, apesar de a probabilidade prevista de entrega de EEE em fim de vida ser de $71 \%$, a composição da amostra e o facto de o questionário ser baseado na expressão de um comportamento e não na revelação do mesmo podem induzir uma sobrestimação da probabilidade de entrega. Assim, podese concluir que em Portugal existe um longo caminho a percorrer de forma a tornar o mercado de retoma de REEE competitivo e atraente para a criação de novos negócios. Por outro lado, os consumidores de EEE ainda se encontram pouco consciencializados para a entrega dos seus REEE, tornando, deste modo, o mercado instável e pouco disponível para usufruir dos benefícios de economias de escala. Consequentemente, sugere-se um estudo exaustivo do público-alvo das campanhas de retoma com o objetivo de direcionar, eficazmente, os incentivos às suas necessidades e motivações. A nível de estrutura de mercado, e comparando com a estrutura existente em outros Estados-Membros, recomenda-se um estudo aprofundado do nível concorrencial existente entre as duas Entidades Gestoras, promovendo uma estrutura de custos de financiamento mais vantajosa e impulsionadora do eco-design.

\section{REFERÊNCIAS}

Atasu A., Subramanian R. (2012). Extended producer responsibility for e-waste: Individual or collective producer responsibility? Production and Operations Management Society 21(6) 1042-1059. doi: 10.1111/j.1937-5956.2012.01327.x

Carvalho M. (2008). Avaliação da situação nacional em matéria de gestão de REEE. Dissertação de Mestrado em Engenharia do Ambiente. Universidade Nova de Lisboa, Portugal

Diretiva n.o 2012/19/UE - Relativa aos resíduos de equipamentos elétricos e eletrónicos. Jornal Oficial da União Europeia, 4 julho

EC (2014). Development of guidance on extended producer responsibility (ERP) - Final Report. European Commission - DG Environment

Ferreira C. (2015). Análise da estrutura de mercado de Resíduos de Equipamentos Elétricos e Eletrónicos - REEE em Portugal. Dissertação de Mestrado em Economia. Universidade do Minho. Braga, Portugal

Gui L., Atasu A., Ergun O., Toktay L.B. (2013). Implementing extended producer responsibility legislation: A multi-stakeholder case analysis. Journal of Industrial Ecology 17(2) 262-276. doi: 10.1111/j.1530-9290.2012.00574.x

Kalimo H., Lifset R., Atasu A., Van Rossem C., Van Wassenhove L. (2015). What roles for which stakeholders under extended producer responsibility? RECIEL: Review of European, Comparative \& International Environmental Law 24(1) 40-57. doi: 10.1111/reel.12087

Kunz N., Atasu A., Mayers K., Wassenhove L. (2014). Extended producer responsibility: Stakeholder concerns and future developments. A white paper prepared by 
INSEAD Social Innovation Centre with the support of ERP - The European Recycling Society

Lundgren K. (2012). The global impact of e-waste: Addressing the challenge. International Labour Organization

Marçal A. (2013). Caracterização e separabilidade com base na forma das partículas de polímeros de REEE. Dissertação de Mestrado em Engenharia do Ambiente Instituto Superior Tecnico, Lisboa, Portugal

Niza S., Santos E., Costa I., Ribeiro P., Ferrão P. (2014). Extended producer responsability policy in Portugal: A strategy towards improving waste management performance. Journal of Cleaner Production 64(1) 277-287. doi: 10.1016/j.jclepro.2013.07.037

Premalatha M., Abbasi-Tabassum, Abbasi T., Abbasi S.A. (2014). The generation impact and management of e-waste: State of the art. Critical Reviews in Environmental Science and Technology 44(14) 1577-1678. doi: 10.1080/10643389.2013.782171

Toyasaki F., Boyaci T., Verter V. (2009). An analysis of monopolistic and competitive take-back schemes for weee recycling. Production and Operations Management 20(6) 805-823. doi: 10.1111/j.1937-5956.2010.01207.x

UNEP (2012). WEEE "take back system". Manual 3. United Nations Environment Programme

Wang Z., Zhang B., Yin J., Zhang X. (2011). Willingness and behavior towards e-waste recycling for residents in Beijing city, China. Journal of Cleaner Production 19(910) 977-984. doi: 10.1016/j.jclepro.2010.09.016

Widmer R., Oswald-Kraf H., Sinha-Khetriwal D., Schnellmann M., Boni H. (2005). Global perspectives on e-waste. Environmental Impact Assessment Review 25(5) 436458. doi: 10.1016/j.eiar.2005.04.001

Yin J., Gao Y., Xu H. (2013). Survey and analysis of consumers' behaviour of waste mobile phone recycling in China. Journal of Cleaner Production 65(15) 517-525. doi: $10.1016 /$ j.jclepro.2013.10.006 